\title{
A Patient with Malignant Spinal Epidural Lymphoma with Initial Rapidly Aggravating Paraplegia
}

\section{Taihei Go, Yasuaki Iida, Hideyuki Aoki, Shintaro Tsuge, Keiji Hasegawa, Yuichiro Yokoyama, Akihito Wada, Yuri Akishima-Fukasawa, Hiroshi Takahashi}

Department of Orthopedics, Toho University, Tokyo, Japan

Email: taiheigo@gmail.com

How to cite this paper: Go, T., Iida, Y., Aoki, H., Tsuge, S., Hasegawa, K., Yokoyama, Y., Wada, A., Akishima-Fukasawa, Y. and Takahashi, H. (2017) A Patient with Malignant Spinal Epidural Lymphoma with Initial Rapidly Aggravating Paraplegia. Open Journal of Orthopedics, 7, 90-97. https://doi.org/10.4236/ojo.2017.73011

Received: February 21, 2017

Accepted: March 28, 2017

Published: March 31, 2017

Copyright $\odot 2017$ by authors and Scientific Research Publishing Inc. This work is licensed under the Creative Commons Attribution International License (CC BY 4.0).

http://creativecommons.org/licenses/by/4.0/

\begin{abstract}
We report the case of a 51-year-old female with rapid neurological deterioration as an initial presentation of non-Hodgkin's lymphoma. Paraplegia occurred suddenly after a 4-day history of weakness and numbness of the lower extremity. MRI revealed a dorsal epidural mass from T10 to T11 that compressed the spinal cord. There was neither bone destruction nor a paravertebral mass. Emergency decompressive laminectomy and tumor resection were performed. Histological analysis of the surgical specimen indicated diffuse large B cell lymphoma. The clinical stage was IV on CT and complete remission was achieved by subsequent chemotherapy. Spinal cord compression occurs in the course of non-Hodgkin's lymphoma in $0.1 \%-6.5 \%$ of cases, but this situation usually develops in the late phase with bone destruction and/or a paravertebral mass. Cord compression and especially the severe symptoms such as paraplegia are rare as the initial presentation of lymphoma.
\end{abstract}

\section{Keywords}

Malignant Lymphoma, Epidural Mass, Paraplegia, Thoracic Spine

\section{Introduction}

Epidural tumors account for about $9 \%$ of malignant spinal epidural lymphomas, and spinal epidural lesions are observed during the course in $0.1 \%-6.5 \%$ of all malignant lymphomas [1]. However, many cases develop as spinal epidural metastasis of a malignant lymphoma, and cases presenting with initial spinal epidural lesions are rare. Symptoms of malignant spinal epidural lymphoma-induced spinal cord compression progress within days to weeks, which make early definite diagnosis and treatment important. A few studies have suggested that ma- 
lignant spinal epidural lymphoma shows characteristic MRI findings, but difficulty with imaging diagnosis has also been pointed out. We encountered a patient with malignant spinal epidural lymphoma that initially presented as rapidly aggravating paraplegia, and we investigated the MRI findings. Here, we report the case with a literature review.

\section{Case Report}

The patient was a 51-year-old woman with a chief complaint of numbness and muscle weakness of the bilateral lower limbs. Her medical history included resection of meningioma (left temporal region) at 44 years old. She had an allergy to iodine contrast agent. Low back pain had developed without a known cause one week before admission. Numbness appeared in the left lower limb 2 days before admission and the patient visited an orthopedic clinic. A mass lesion at the T10-11 level on thoracic spinal MRI was observed on the next day, and the patient was referred to our hospital for close examination and treatment. The first examination showed numbness of the bilateral lower limbs that was severer on the left side and pain of the anterior surfaces of the bilateral thighs. Walking was possible, but listlessness of the bilateral lower limbs was observed. Thus, the patient was admitted on the same day.

At admission, anesthesia of sense of vibration was noted at L1 or lower, but thermal nociception and sense of optimism were normal. Tendon reflexes were slightly promoted in the bilateral lower limbs, and muscle weakness of the lower limbs (right: MMT 4, left: MMT 3-4) was present. There was no bladder or rectal disturbance. Since progressive aggravation of spinal cord symptoms was observed, a re-examination by MRI was performed on the day of admission. A mass lesion was found on the dorsal side of the spinal dural canal at T10-11, and findings indicating thoracic spinal cord compression were observed. The tumor was homogeneous inside and showed isointensity on T1-weighted imaging and slightly high intensity on T2-weighted imaging, compared with the spinal cord (Figure 1).

There was no bone destruction of the vertebral body or paravertebral mass. These findings were not markedly different from those on the previous MRI examination.

Spinal cord symptoms rapidly aggravated to Frankel classification A on day 2 after admission. Therefore, thoracic spinal laminectomy and excision of the spinal epidural mass were urgently performed for diagnosis and treatment. In surgery, laminectomy was applied to T9-11. The bone quality was favorable and no bone lesion was noted. Detachment of the yellow ligament revealed an epidural dark red elastic soft mass rich in blood vessels with an unclear boundary (Figure 2). Adhesion with the dorsal side of the dura mater was mild, and as much as possible of the mass was resected to resolve compression of the thoracic spinal cord. After excision, bulging of the dural canal was favorable and slight pulsation was noted.

Intraoperative histopathological examination of the excised mass showed 


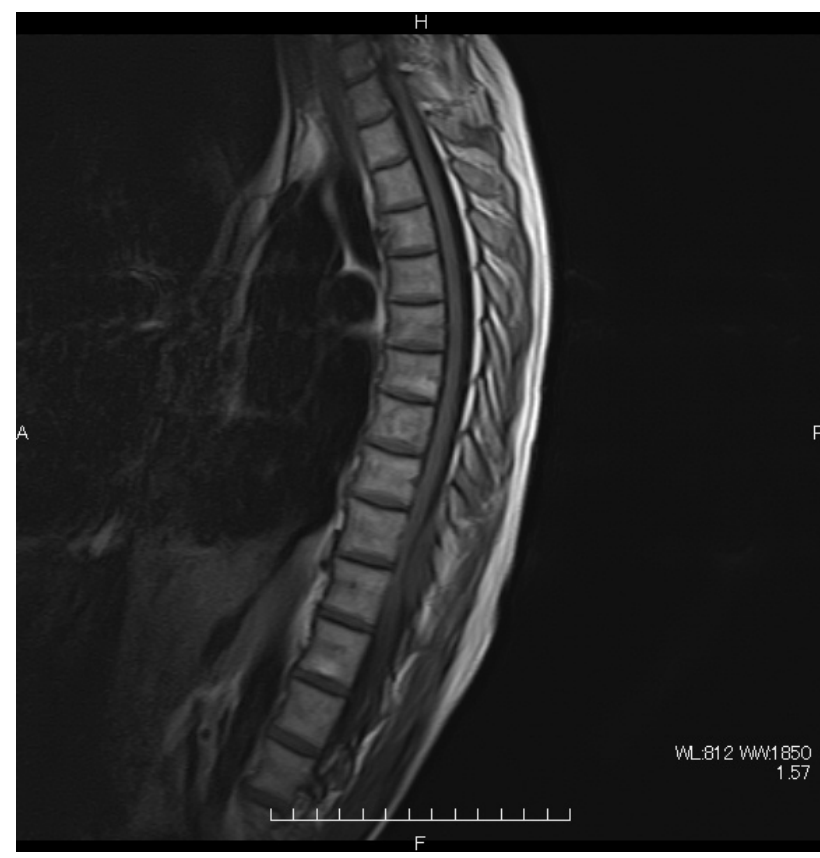

(a)

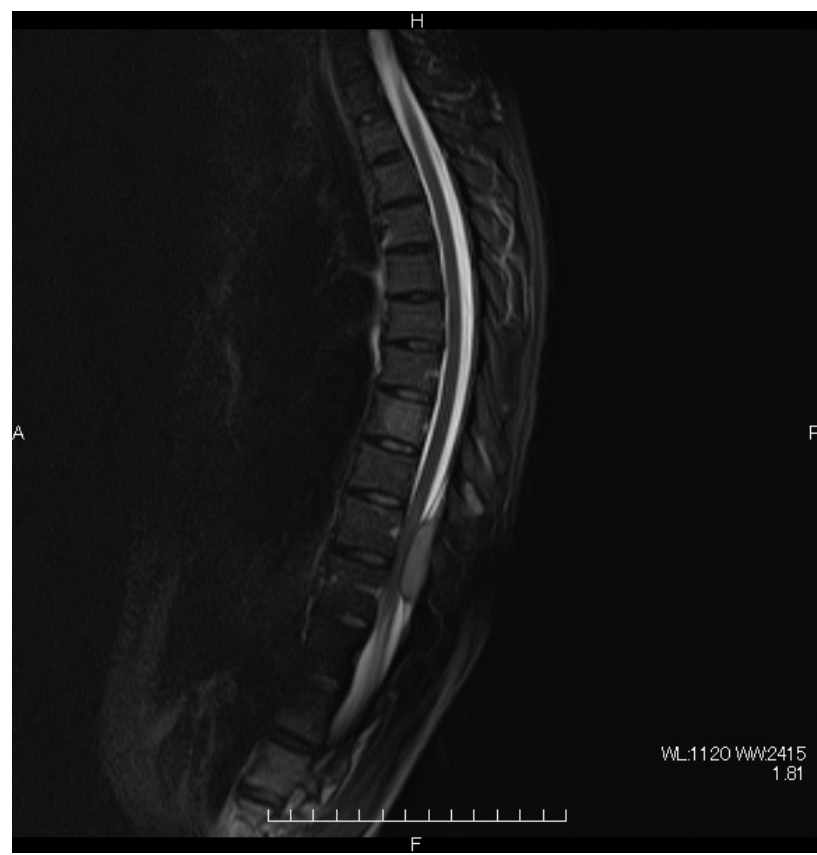

(c)

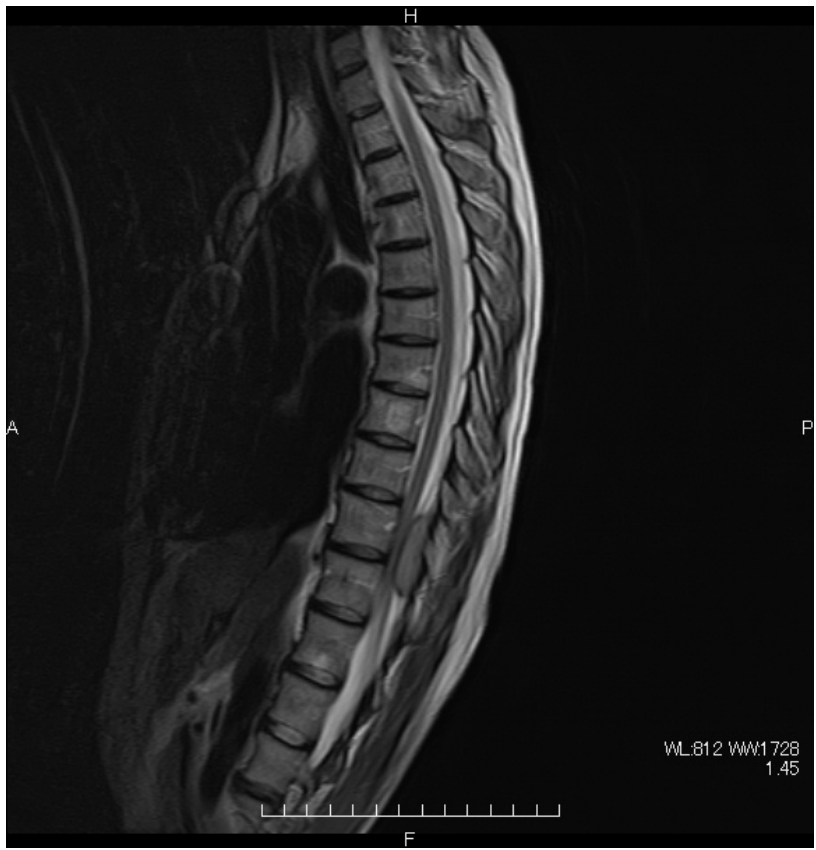

(b)

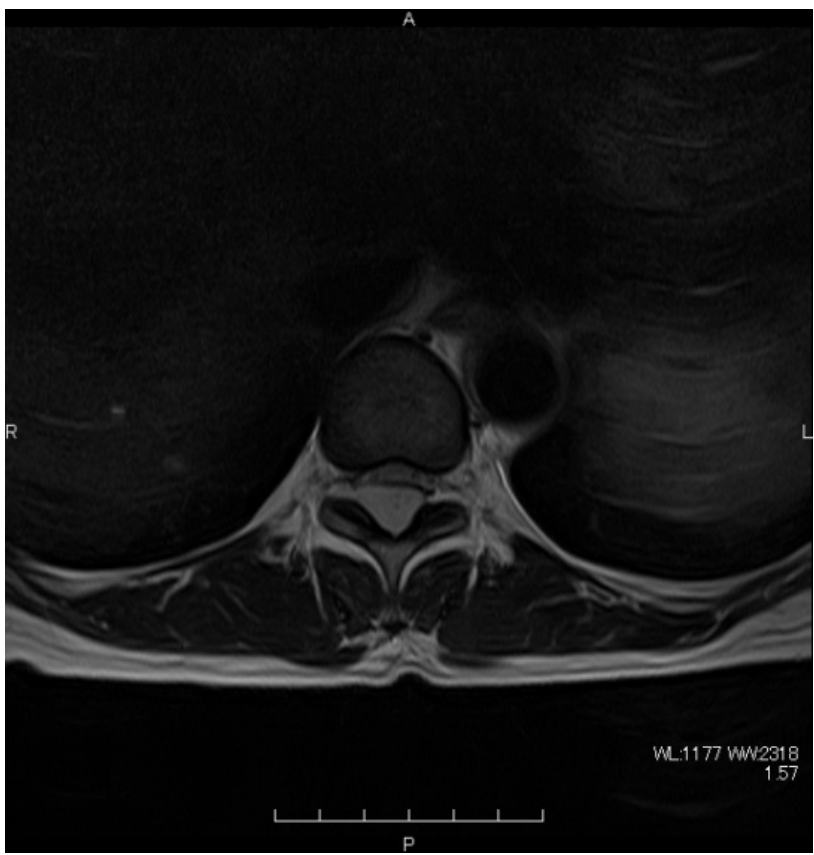

(d)

Figure 1. MRI using (a) sagittal T1-weighted imaging, (b) sagittal T2-weighted imaging, (c) sagittal T2-weighted imaging (STIR), and (d) axial T2-weighted imaging. Mass lesions excluding the dural canal from the spinal epidural dorsal side were present at the T10 - 11 vertebral body level. There was no vertebral body lesion or paravertebral mass, but the spinal epidural lesion advanced into the right intervertebral foramen.

diffusely proliferating medium to large-sized cells. Immunostaining for CD20 and CD79 was positive, suggesting a B-cell tumor. HE staining showed proliferation of medium to large cells, which led to a definite diagnosis of malignant lymphoma (non-Hodgkin type, diffuse large B cell) (Figure 3). The MIB-1 index was high (about 90\%) and features of aggressive proliferation were present, but 


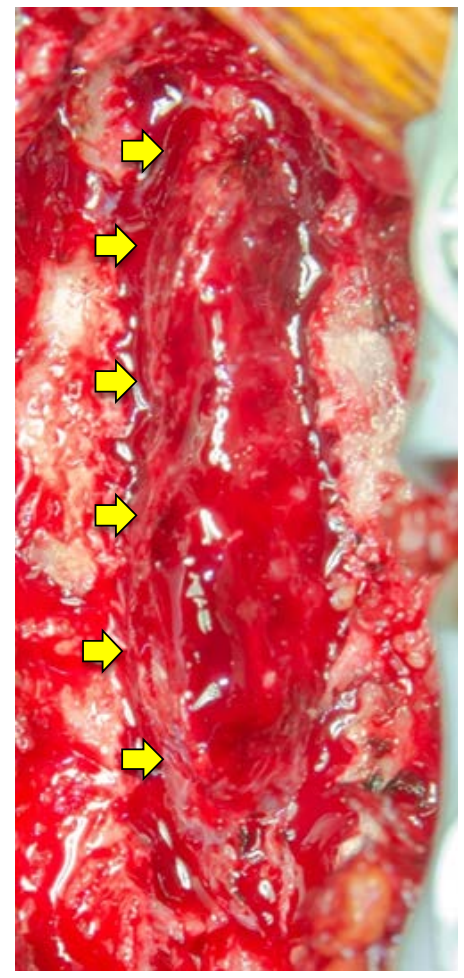

(a)

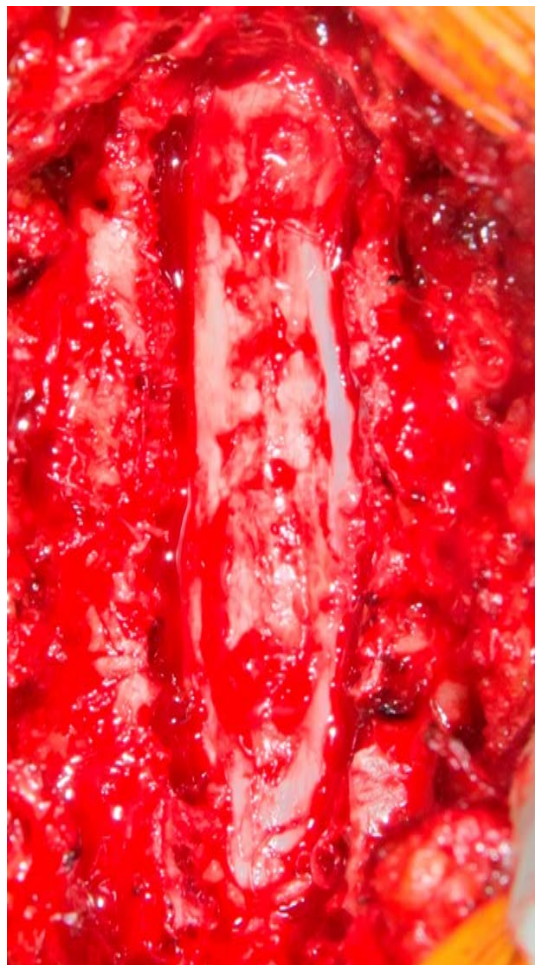

(b)

Figure 2. Intraoperative findings: (a) before tumor excision and (b) after tumor excision. An epidural dark red elastic soft mass rich in blood vessels with an unclear boundary was present. (Yellow arrows pointed to the boundary.) After excision of the mass, bulging of the dural canal was favorable.

bcl-2 was positive and bcl-6 was negative, excluding Burkitt lymphoma. Epithelial membrane antigen (EMA) was negative, excluding meningioma.

After surgery, the patient underwent whole body CT. Lymph node lesions were present in the bilateral hila of the kidneys, and the clinical disease stage was IV. Radiotherapy was not performed and remission was achieved by chemotherapy (RCHOP therapy) alone. Paraplegia improved to a level of walking with crutches one year after surgery, and disturbance of sensation also gradually improved. Bladder and rectal disturbance did not improve initially, but had been resolved by 1.5 year after surgery.

\section{Discussion}

The incidence of malignant spinal epidural lymphoma is high in men in their 50s to 70 s, and patients aged 40 years or older account for more than $80 \%$ of cases [1]. The site is at the thoracic spinal cord level in $75 \%$ of cases [2], which is thought to be due to the thoracic spinal cord being longer than the cervical and lumbar spinal cord, and the presence of abundant venous plexus [3]. Regarding symptoms, lumbar backache precedes diagnosis and persists for several months to about one year and neurologic manifestation aggravate through a subacute course in several weeks [4]. The present case was mostly typical except that the patient was female and the disease course was relatively rapid. Iizuka, et al. roughly 


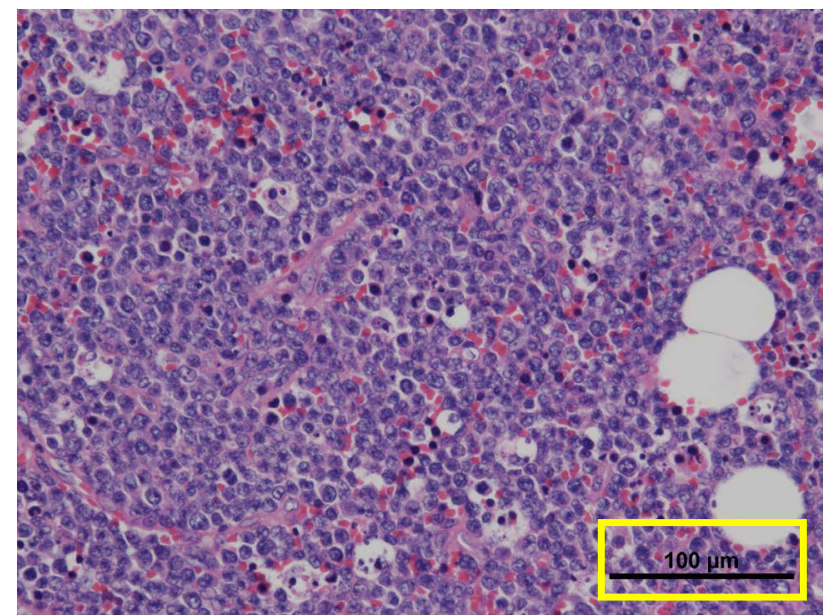

(a)

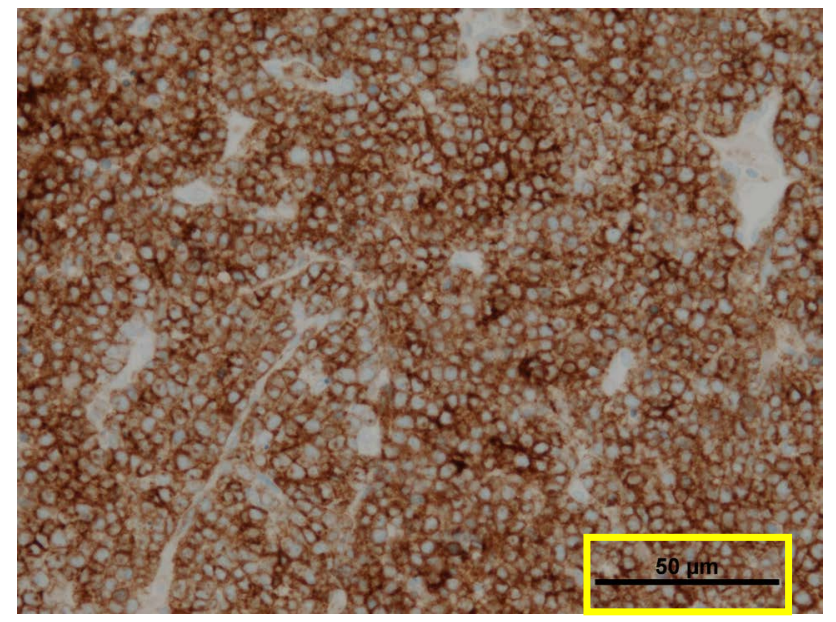

(c)

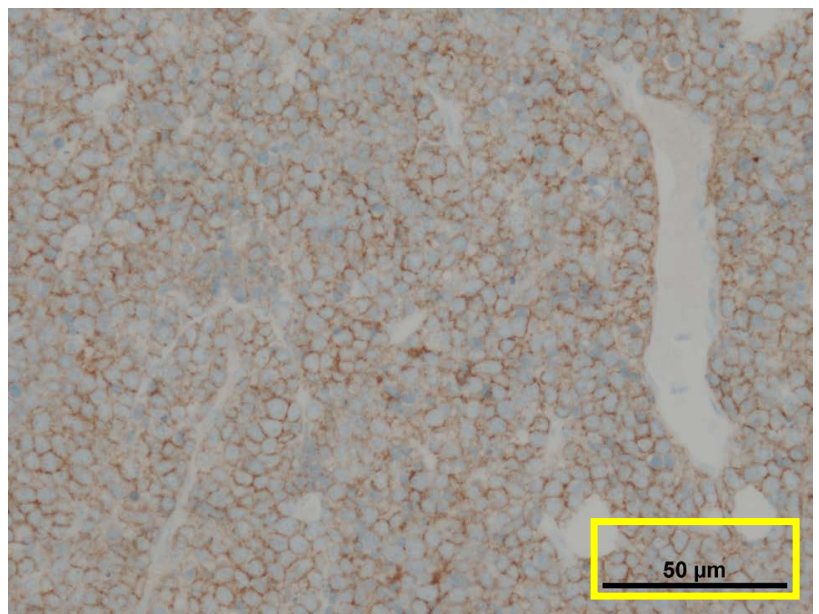

(b)

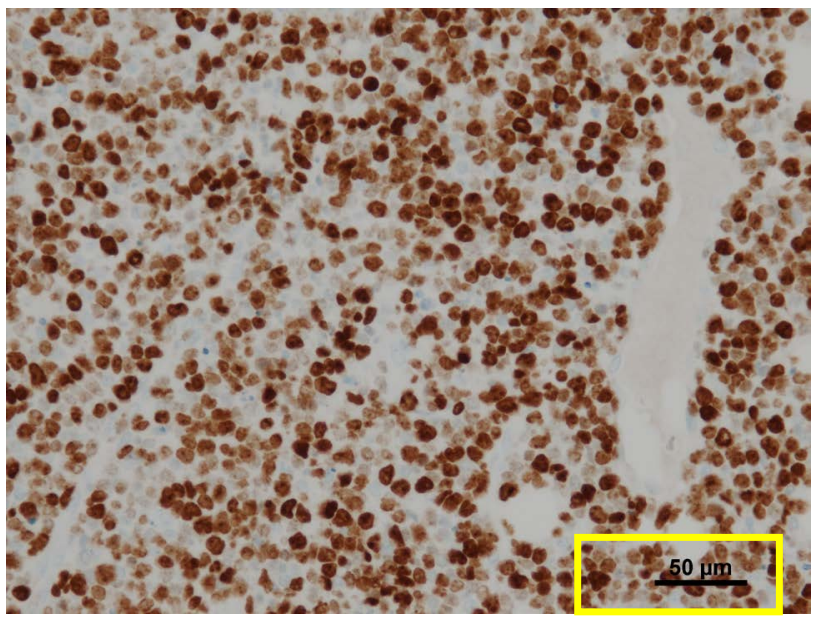

(d)

Figure 3. Histopathological view: (a) HE staining, (b) CD20, (c) CD79, (d) MIB-1. CD20 and CD79 (B cell markers) were positive on immunostaining, and diffuse proliferation of medium to large-sized cells was noted on HE staining, leading to diagnosis of diffuse large B cell lymphoma. The MIB-1 index was high, showing that the tumor had aggressive proliferative properties.

divided malignant spinal epidural lymphomas into cases developing as spinal epidural metastasis of malignant lymphoma and those with initial low back pain and spinal cord symptoms [5]. Our patient had lymph node lesions in the hila of the bilateral kidneys on whole body examination after surgery, suggesting an advanced stage of metastasis, but the patient had not previously been diagnosed with malignant lymphoma and the disease initially developed with spinal cord symptoms.

Primary malignant spinal epidural lymphoma with no other lesion detected in a whole body examination accounts for less than half of non-Hodgkin lymphoma (NHL) cases that initially develop with spinal epidural lesions, and is considered to be a rare disease [1]. However, the presence of lymph tissue in the spinal epidural space is uncertain and has not been shown. Generally, metastasis from an unidentified lesion is possible, such as infiltration from the adjacent paraspinal lymph node and vertebral body. McDonald, et al. classified malignant spinal epidural lymphomas into four groups based on the range of advancement 
[6]: (i) tumors of primary bone origin, (ii) paravertebral tumors, (iii) tumors with bony vertebral destruction and a paravertebral mass, and (iv) tumors with neither bone destruction nor a paravertebral mass. Only (iv) is a primary spinal epidural lymphoma in a pure sense. In our patients, the lesions in the hila of the bilateral kidneys found after surgery indicate a secondary condition, but no vertebral body or paraspinal lesion was detected on imaging, which corresponds to (iv) in the classification above. There was no paraspinal lesion, but the spinal epidural lesion was continuous with the right intervertebral foramen (Figure 1), suggesting an aggressive lymphoma originating from the spinal epidural region, advancing through the intervertebral foramen, and metastasizing in the hila of the kidneys. The patient had a normal life until the development of spinal cord symptoms, with no characteristic systemic symptoms of malignant lymphoma such as fever, night sweat and weight loss. This course may also be consistent with the type (iv) classification.

The characteristic MRI findings of malignant spinal epidural lymphoma are that the tumor has homogeneous content and shows low to isointensity on T1-weighted imaging and isointensity to high intensity on T2-weighted imaging, compared with the intensity of the spinal cord. Homogeneous enhancement is observed on contrast-enhanced MRI, with the lesion appearing long in the vertical direction and extending over several vertebral bodies, advancing from the paraspinal region to the intervertebral foramen in many cases, and being located on the dorsal side of the dural canal in many cases [7] [8]. In cases of infiltration from bone lesions, epidural lesions may be located on the ventral side of the dural canal, diffuse changes in the intensity are observed in the vertebral bone marrow at the level of the lesion, and lesions may be observed at non-continuous spinal cord levels [9]. All these imaging findings are nonspecific, suggesting that it is difficult to make a definite diagnosis using imaging.

In the 572 cases of malignant lymphoma treated at the hematology department of our hospital between 2003 and 2014, none was a malignant spinal epidural lymphoma with initial spinal cord symptoms and without a vertebral body or paravertebral mass. Thus, the case reported here is very rare. About $0.9 \%$ $6.5 \%$ of NHL cases before diagnosis initially develop as a spinal epidural lesion [1], and Perry, et al. found paraplegia in only 3 of 18 patients with malignant spinal epidural lymphoma that initially developed with spinal cord symptoms [10].

The prognosis is better when lesions are localized in the spinal epidural space, and poor in cases of spinal epidural space metastasis [11]. The severity of preoperative spinal cord symptoms also influences the outcome. In Perry, et al., functional recovery was not achieved and the mean life expectancy was $6-7$ months in 3 patients who developed paraplegia before surgery [10]. Our patient presented with paraplegia, but the postoperative course was very good at one year and 3 months. The clinical course was favorable as a clinical disease stage IV case manifesting paraplegia. This suggested that the lesion initially developed in the spinal epidural space and then metastasized to the lymph nodes in the hila 
of the kidneys.

In addition to mechanical compression, spinal cord symptoms may develop due to tumor invasion through the intervertebral foramen occluding the radicular artery and resulting in ischemic changes in the spinal cord [12]. In our patient, bulging of the dural canal and pulsation were achieved by emergency laminectomy and excision of the mass. Rapid emergency surgery may have minimized the effects of mechanical compression and ischemic changes, and chemotherapy may have been effective because the lesion was aggressive.

\section{Conclusion}

We encountered a case of malignant spinal epidural lymphoma that presented with rapidly aggravating paraplegia as the initial symptom. Definite diagnosis of myelopathy caused by epidural lesions is difficult based on imaging findings. In such cases, it is necessary to perform rapid decompression of the spinal cord and a pathological examination.

\section{Consent}

The patient gave informed consent to submit this case study for publication.

\section{Competing Interests}

The authors declare that there is no conflict of interest regarding the publication of this paper.

\section{References}

[1] Cugati, G., et al. (2011) Primary spinal Epidural Lymphomas. Journal of Craniovertebral Junction and Spine, 1, 3-11. https://doi.org/10.4103/0974-8237.85307

[2] Monnard, V., et al. (2006) Primary Spinal Epidural Lymphoma: Patients' Profile, Outcome, and Prognostic Factors: A multicenter Rare Cancer Network study. International Journal of Radiation Oncology, Biology, Physics, 65, 817-823. https://doi.org/10.1016/j.ijrobp.2006.01.002

[3] Mora, J. and Wollner, N. (1999) Primary Epidural Non-Hodgkin Lymphoma: Spinal Cord Compression Syndrome as the Initial Form of Presentation in Childhood Non-Hodgkin Lymphoma. Medical and Pediatric Oncology, 32, 102-105. https://doi.org/10.1002/(SICI)1096-911X(199902)32:2<102::AID-MPO6>3.0.CO;2$\underline{\mathrm{Y}}$

[4] Epelbaum, R., et al. (1986) Non-Hodgkin's Lymphoma Presenting with spinal Epidural Involvement. Cancer, 58, 2120-2124.

https://doi.org/10.1002/1097-0142(19861101)58:9<2120::AID-CNCR2820580926>3. 0.CO;2-A

[5] Iizuka, H., et al. (1989) Malignant Lymphoma of the Spinal Epidural Space: Report of three Cases. Neurologia medico-chirurgica, 29, 307-311. https://doi.org/10.2176/nmc.29.307

[6] McDonald, A.C., Nicoll, J.A. and Rampling, R.P. (2000) Non-Hodgkin's Lymphoma Presenting with Spinal Cord Compression; a Clinicopathological Review of 25 Cases. European Journal of Cancer, 36, 207-213. https://doi.org/10.1016/S0959-8049(99)00265-8 
[7] Nakayama,T., Miki, Y. and Ehara, S. (2010) Approach to Spinal Cord and Spinal Diseases: Epidural Lesions. Japanese Journal of Clinical Radiology, 55, 289-319.

[8] Mascalchi, M., et al. (1995) MRI of Spinal Epidural Lymphoma. Neuroradiology, 37, 303-307. https://doi.org/10.1007/BF00588341

[9] Boukobza, M., et al. (1996) Primary Vertebral and Spinal Epidural Non-Hodgkin's Lymphoma with Spinal Cord Compression. Neuroradiology, 38, 333-337. https://doi.org/10.1007/BF00596582

[10] Perry, J.R., et al. (1993) The Significance of Spinal Cord Compression as the Initial Manifestation of Lymphoma. Journal of Neurosurgery, 32, 157-162. https://doi.org/10.1227/00006123-199302000-00001

[11] Perry, J.R., Deodhare, S.S., Bilbao, J.M., Murray, D. and Muller, P. (1992) Primary spinal Epidural Non-Hodgkin's Lymphoma: Report of Eight Patients and Review of the Literature. Journal of Neurosurgery, 30, 675-680. https://doi.org/10.1227/00006123-199205000-00004

[12] Mullins, G.M., Flynn, J.P., El-Mahdi, A.M., McQueen. J.D. and Owens, A.H.J. (1971) Malignant Lymphoma of the Spinal Epidural Space. Annals of Internal Medicine, 74, 416-423. https://doi.org/10.7326/0003-4819-74-3-416

Submit or recommend next manuscript to SCIRP and we will provide best service for you:

Accepting pre-submission inquiries through Email, Facebook, LinkedIn, Twitter, etc. A wide selection of journals (inclusive of 9 subjects, more than 200 journals) Providing 24-hour high-quality service User-friendly online submission system Fair and swift peer-review system Efficient typesetting and proofreading procedure Display of the result of downloads and visits, as well as the number of cited articles Maximum dissemination of your research work

Submit your manuscript at: http://papersubmission.scirp.org/

Or contactojo@scirp.org 